\title{
Small bowel intussusception in a child following feeding jejunostomy: A rare case with review of literature
}

\author{
Basant Kumar ${ }^{1 *}$, Richa Lal ${ }^{1}$, Vijai Datta Upadhyaya ${ }^{1}$, Banani Poddar ${ }^{2}$ \\ ${ }^{1}$ Department of Paediatric Surgery, Sanjay Gandhi Post Graduate Institute of Medical Sciences, Lucknow, India \\ ${ }^{2}$ Department of Critical Care Medicine, Sanjay Gandhi Post Graduate Institute of Medical Sciences, Lucknow, India \\ Email: *drbkpg75@rediff.com, drbasant189@gmail.com
}

Received 26 April 2012; revised 25 July 2012; accepted 21 November 2012

\begin{abstract}
Jejuno-jejunal intussusception is a rare complication of feeding jejunostomy tube placement. A case of one year old child who underwent gastric pull-up for complicated tracheo-esophageal fistula had jejunojejunal intussusception induced by Witzel's feeding jejunostomy tube; is discussed with review of literature.
\end{abstract}

Keywords: Jejuno-Jejunal Intussusception; Feeding Jejunostomy; Tracheo-Esophageal Fistula

\section{INTRODUCTION}

Small bowel intussusception is a rare complication of feeding jejunostomy tube placement. It is characterised by transient small bowel obstruction due to small bowel segment intussusceptions and usually resolves spontaneously. Most of the times; enteral feeding can be continued without difficulty. But in few cases reported in literature, operative intervention is required to relieve bowel obstruction. There is some lack of literature for its preoperative diagnosis and surgical treatment especially in infants and children. Here, we present our experience with jejuno-jejunal intussusception following Witzel's feeding jejunostomy after gastric pull-up in a child with complicated trachea-esophageal fistula and review the literature.

\section{CASE REPORT}

One year old male child weighing $10 \mathrm{~kg}$ was admitted as a follow-up case of trecheo-esophageal fistula with decompressive gastrostomy and feeding jejunostomy, for definitive procedure (gastric pull-up). He was operated for tracheo-esophageal fistula on 2nd day of life and developed esophageal stricture after 1.5 months of surgery. Stricture was initially managed by endoscopic dilatation (6 - 7 sessions). But it got complicated by esophageal per-

"Corresponding author. foration with severe mediastinitis during dilatation at 3 months of age. Emergency end-esophagostomy with transthoracic mediastinal drainage with decompressive gastrostomy and Witzel's feeding jejunostomy was performed at that time.

During definitive procedure, a retro-sternal gastric pullup was performed. Witzel's feeding jejunostomy was left in-situ and a naso-gastric tube was placed to keep the intrathoracic stomach decompressed. He tolerated jejunostomy feeds well initially, but, was noticed to have high volume bilious aspirates via the nasogastric tube on the 10th postoperative day. The day after, he vomited and aspirated for which he had to subjected to an emergency tracheal intubation. A contrast upper gastro-intestinal study through naso-gastric tube and feeding-jejunostomy tube was performed which showed dilatated proximal bowel with non-progression of the contrast medium into the distal bowel. Diagnosis was further confirmed by bed side ultrasonography which showed "target” lesion. Emergency re-exploration was done. About $15 \mathrm{~cm}$ long, antegrade small bowel intussusception, distal to jejunostomy-site with dilated proximal jejunal loops was present. Reduction of intussusception was done. There was no evidence of gangrene. Feeding jejunostomy was left in-situ. Gradual jejunostomy feed was restarted from the 3rd postoperative day onwards following reduction of intussusception and was well tolerated. Feeding jejunostomy was removed after 6 weeks. In the last 1 year of follow-up, child had no complaints with good activity and oral acceptance of feeds (Figure 1).

\section{DISCUSSION}

Enteral feeding is the preferred route for nutritional support because of its trophic effects on the gastrointestinal tract with less bacterial translocation and low incidence of infective complications [1]. It is more physiological and cost effective than the parental route. Gastrostomy, gastro-jejunostomy, jejunostomy and naso-jejunal tube placement gives a temporary access to the gastrointestinal tract for enteral feeding. It can be done by open tech- 


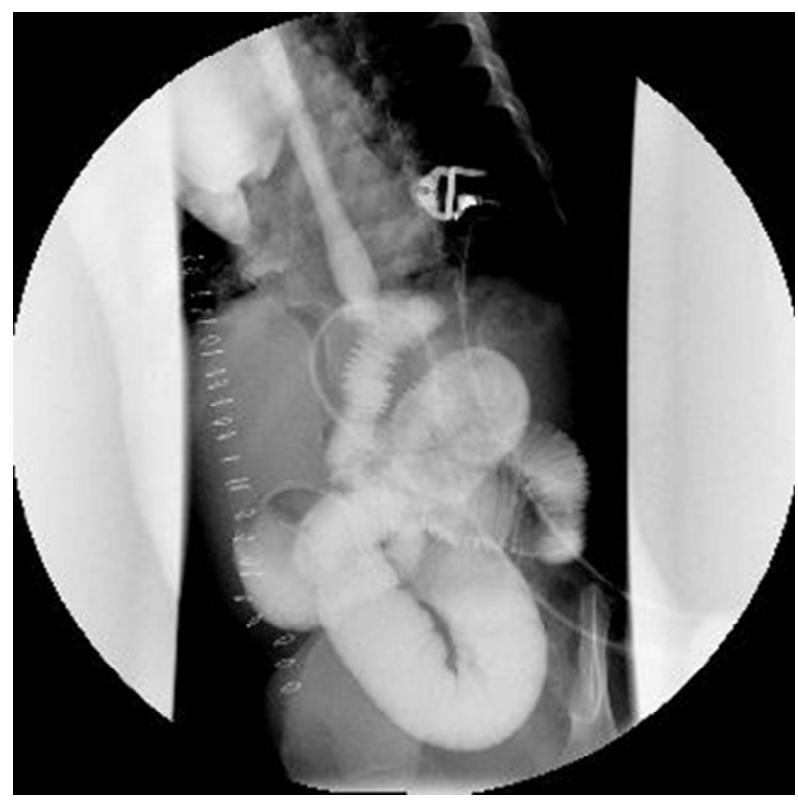

Figure 1. Contrast study through Naso-Jejunal tube and feedingjejunostomy tube showing dilated proximal bowel and obstruction at the tip of jejunostomy tube.

nique or by less invasive techniques like endoscopic, fluoroscopic and laparoscopic [2]. Various complications of tube jejunostomy are described in literature including mechanical complications, infections, gastrointestinal problems and metabolic abnormalities [3]. These complications may be major, requiring significant medical or surgical interventions, or it may be minor ones. It may occur early (within 30 days) or late. Major complications are less frequent after radiologic gastrostomy (5.9\%) compared with surgical gastrostomy (19.9\%) [3].

Although jejunostomy tube induced small bowel intussusception is a rare complication, cases are reported in literature. Actual incidence of this problem is unknown. Most of the instances of this complication reported in literature are related to the adult population. Carucci, L.R., et al. [2] reported 4 cases (1\%) of small bowel intussusception at the site of jejunostomy tube but none of them needed operative intervention and resolved spontaneously. Friedman, J.N., et al. [3] described 8\% of intussusceptions in patients with GJ tube inserted. Wales, P.W., et al. [4] reported that obstruction in patients was secondary to intussusceptions around the gastro-jejunostomy tube (20.8\%). No patient required laparotomy and intussusception was treated by shortening of feeding tube. 30\% cases of intussusceptions occurred within 30 days of tube insertion [4]. Connolly, B.L., et al. [5] observed 7 intussusceptions in 5 children with gastro-jejunostomy tubes. The intussusceptions were often transient or intermittent. The usual presentations were vomiting (often bilious); intolerance to feed and some were entirely asymptomatic. Patients rarely presented with abdominal pain or with classical features of intussusception. Predisposing factors seem to include male gender, young infants and the presence of distal pigtail on the tube [6]. The diagnosis of intussusceptions around tube is not easy as it may not interfere with the tube feeding. The clinical symptoms and findings on plain abdominal radiographs and in upper gastrointestinal contrast study may not be much different from other causes of small bowel obstruction. Therefore, the diagnosis of intussusception will be delayed [7]. Connolly, B.L., et al. [5] reported the use of sonography as well as fluoroscopy in the diagnosis of the intussusceptions in these cases.

The exact aetiology and mechanism of intussusceptions induced by jejunostomy tube is unclear. Connolly, B.L., et al. [5] hypothesized that the loop of the pigtail acts as a lead point, dragging the bowel with it. The other hypothesis is that the tube sets up a chronic inflammatory reaction in the bowel and the hypertrophied mucosa creates a lead point for intussusceptions. Wu, T.H., et al. [7] suggested that retrograde peristalsis of jejunum during the vomiting episodes and an injecting force produced by tube feeding with pump infusion to the jejunostomy tube; which acts as stent, is the cause of intussusceptions. Thin built of patient also favours intussusception because of small amount of fatty tissue (omentum, mesentery) which allows small bowel to move more freely in the abdominal cavity [7].

Although most of the times, jejunostomy tube induced intussusceptions resolves spontaneously, sometimes they need operative intervention as in our case. Most intussusceptions can be managed successfully by replacing the gastro-jejunostomy tubes with standard or shortened tubes without distal pigtail [3]. Reduction of the intussusceptions can also be achieved by bolus injection of air or contrast medium through the tube or exchange over a wire under fluoroscopy [5]. As radiologic reduction by hydrostatic/contrast or air enema are less successful with uncertain results [8] and our patient was critically ill and was on ventilator; we decided to re-explore rather than rely on expectant therapy. There was no role of laparoscopic reduction as it was a post-operative case. Preoperative CT-scan was not done. Operative reduction of intussusceptions without resection gave good result. Although the jejunostomy tube is the cause of intussusception, it was not removed during the operation; as done by Wu, T.H., et al. [7]. Child had the same feeding tube from last 10 months without any complication but after gastric pull-up, he developed this problem; we could not explain the cause of it. We could not find any literature on small bowel intussusceptions following Witzel's feeding jejunostomy in a child operated as gastric pull-up for trachea-esophageal fistula. Also, we could not find any literature/study comparing this complication in different types of jejunostomies. 
In conclusion, this case emphasises the need of high degree of suspicion to diagnose small bowel intussusception in patients with jejunostomy tube, having gastrointestinal symptoms. Upper gastro-intestinal contrast study and abdominal sonography is sufficient for diagnosis. Laparotomy was strongly indicated in patients with persistent sign and symptoms, as in our case. Operative reduction is sufficient and resection is not advised if there are no gangrenous changes or perforation. It is not necessary to remove the jejunostomy tube and it can be used postoperatively without recurrence of intussusception [7].

\section{REFERENCES}

[1] Gottardi, A.D., Krahenbuhl, L., Farhadi, J., Gernhardt, S., Schafer, M. and Buchler, M.W. (1999) Clinical experience of feeding through a needle catheter jejunostomy after major abdominal operations. European Journal of Surgery, 165, 1055-1060. doi:10.1080/110241599750007892

[2] Carucci, L.R., Levine, M.S., Rubesin, S.E., Laufer, I., Assad, S. and Herlinger, H. (2002) Evaluation of patients with jejunostomy tubes: Imaging findings. Radiology, 223, 241-247. doi:10.1148/radiol.2231010961

[3] Friedman, J.N., Ahmad, S., Connolly, B., Chait, P. and
Mahant, S. (2004) Complications associated with imageguided gastrostomy and gastrojejunostomy tubes in children. Pediatrics, 114, 458-461. doi:10.1542/peds.114.2.458

[4] Wales, P.W., Diamond, I.R., Dutta, S., Muraca, S., Chait, P., et al. (2002) Fundoplication and gastrostomy verses image-guided gastrojejunal tube for enteral feeding in neurologically impaired children with gastroesophageal reflux. Journal of Pediatric Surgery, 37, 407-412. doi:10.1053/jpsu.2002.30849

[5] Connolly, B.L., Chait, P.G., Siva-Nandan, R., Duncan, D. and Peer, M. (1998) Recognition of intussusceptions around gastrojejunostomy tubes in children. American Journal of Roentgenology, 170, 467-470.

[6] Hughes, U.M., Connolly, B.L., Chait, P.G. and Muraca, S. (2000) Further report of small bowel intussusceptions related to gastrojejunostomy tubes. Pediatric Radiology, 30, 614-617. doi:10.1007/s002470000260

[7] Wu, T.H., Lin, C.W. and Yin, W.Y. (2006) Jejunojejunal intussusceptions following jejunostomy. Journal of the Formosan Medical Association, 105, 355-358. doi:10.1016/S0929-6646(09)60129-7

[8] Parikh, M., Samujh, R., Kanojia, R. and Sodhi, K.S. (2010) Does all small bowel intussusception need exploration. African Journal of Paediatric Surgery, 7, 30-32. 